\title{
Structure and properties of polymers
}

\author{
A. ZIABICKI \\ Institute of Fundamental Technological Research \\ Polish Academy of Sciences \\ ul. Świętokrzyska 21, 00-049 Warszawa, Poland \\ aziab@ippt.gov.pl
}

\begin{abstract}
A characteristic feature of polymer materials is structure-dependent physical properties. Structure on many different levels - from interatomic and molecular distances to microscopic structure - strongly affect mechanical, thermal, and optical behaviour. Consequently, a variety of properties and uses can be obtained from materials of the same chemical composition by development of structure in thermal and mechanical treatment. Mathematical models describing various structures based on thermodynamic and kinetic considerations are developed and compared with experimental data.

First part of this paper presents different levels of polymer structure. Part two is concerned with a special class of polymers - rubbers. Rubber properties are controlled by topological structure of crosslinked polymers - systems in which linear molecular chains are connected into a coherent system. Part three presents generalized theory of crystal nucleation. Crystallization of polymers is very sensitive to external fields (hydrodynamic, electrostatic, electromagnetic, gravitational, etc.). Analysis of field effects required introduction of new variables into the classical nucleation theory. The outcome of the generalized theory was, int. al., new nucleation mechanisms like rotation (translation) in a potential field or "healing" of defective internal structure.
\end{abstract}

Key words: polymer, structure levels, polymer crystals, molecular orientation, rubbers, rubber elasticity, polymer networks, crosslinking, topological structure of networks, nucleation of phase transitions, thermal nucleation, athermal nucleation.

\section{Polymer materials}

\subsection{Polymers vs. metals and ceramics}

One of the basic properties of organic polymers is wide variety of physical properties at the same chemical composition. When classical materials metals, alloys or ceramics are considered - it is chemical composition which controls properties and uses. When we find that an iron alloy contains $3 \%$ carbon, $0.5 \%$ manganese, $2 \%$ silicon and $0.3 \%$ phosphorous - we can say for 
sure that it is cast iron - inexpensive, rather brittle construction material for common uses. Iron alloy containing $1 \%$ carbon, $12 \%$ wolfram (tungsten) and small additions of chromium, manganese and vanadium, is a high-quality tool steel.

Properties of organic polymers, on the other hand, depend as much on chemical composition as on molecular and supramolecular structure on several levels. Consider a popular hydrocarbon, a linear chain consisting of repeating units $-\mathrm{CH}_{2}-\mathrm{CH}_{2}-$. Chemically identical materials containing ca. $86 \%$ of carbon and $14 \%$ of hydrogen may have very different properties.

Molecule of a popular hydrocarbon called ethylene $\mathrm{CH}_{2}=\mathrm{CH}_{2}$ with molecular mass $M_{w}=28 \mathrm{da}$ ) is a gas. Somewhat larger molecule, a chain composed of five ethylene units terminated with hydrogen atoms, decane, $\mathrm{H}-\left(\mathrm{CH}_{2}-\mathrm{CH}_{2}\right)_{5}-\mathrm{H}$ (molecular mass $M_{w}=142 \mathrm{da}$ ) is a mobile liquid. Lowmolecular-weight polymer chemically identical with decane, polyethylene $\left(M_{w}=10,000 \mathrm{da}\right)$ has properties of wax, common grade polyethylene $\left(M_{w}=100,000-200,000 \mathrm{da}\right)$ is a popular construction and packaging material, while ultra-high-molecular-weight polyethylene $\left(M_{w}>1,000,000 \mathrm{da}\right)$ is used for manufacturing ultra-strong fibers for reinforcement of composites, and bullet-proof vests. All the above materials have identical chemical structure and composition. The only difference consists in molecular mass.

\subsection{Structure levels}

Let us analyze different levels of structure and discuss its effect on material properties. Structure levels cover wide range of $0.1-10^{4} \mathrm{~nm}$, from molecular groups consisting of several atoms to large supramolecular forms exceeding one micrometer.

\subsubsection{Molecular structure $(0.1-10 \mathrm{~nm})$}

\section{Simple inorganic materials}

Metals, oxides, ceramics - consist of small molecules $\left(M_{w}=10-1000 \mathrm{da}\right)$ with strong intra-, and intermolecular bonds. Physical properties are to a large extent controlled by chemical composition.

\section{Organic polymers}

Polyethylene, polyamides, polyesters - present long macromolecules composed of hundreds or thousands of repeating units (mers) with molecular mass $M_{w}=5 \times 10^{3}-5 \times 10^{6} \mathrm{da}$. The same chemical composition can be accompanied by different structures and properties. The main factors which 
control properties include:

- chemical structure,

- average molecular mass, $M_{w}$,

- molecular mass distribution, $\mathrm{f}\left(\mathrm{M}_{w}\right)$

- defects of polymer chains,

- texture and supramolecular structure associated with thermal history. Chemical structure of polymers can be modified by: copolymerization - synthesis of polymers composed of different chemical units arranged in different order and proportions, and attachment of active chemical groups which can convert a polymer into a dyestuff, a drug, optically active center, or electric conductor.

Figure 1 presents molecular model of a typical polymer (polyethylene). The black backbone consists of carbon atoms, white balls represent hydrogens. Dimensions are represented in the $5 \times 10^{7}: 1$ scale. Figure 2 shows scheme of the same molecule indicating main chemical bonds $(\mathrm{C}-\mathrm{C})$ capable of rotation.

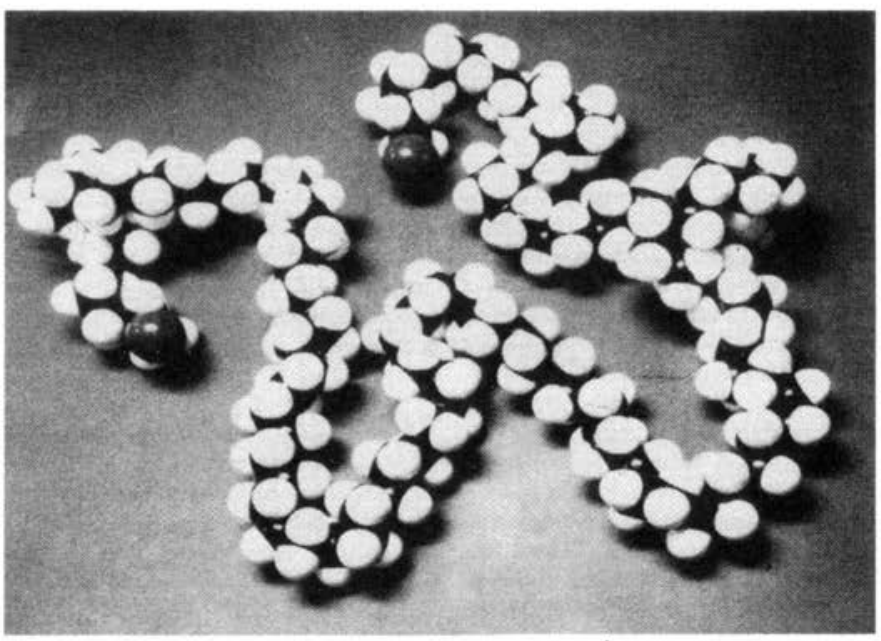

Figure 1. Atomic model of a polyethylene chain.

The fact that polymer molecules ("macromolecules") consist of many repeating units connected with chemical bonds leads to features, specific for polymers and absent in inorganic, low-molecular-weight materials:

1. Many internal (rotational) degrees of freedom make from each macromolecule a statistical system with variable entropy.

2. Energy of intermolecular interactions is a sum of interactions of all repeating units and is proportional to (very large) molecular weight $M_{w}$. 


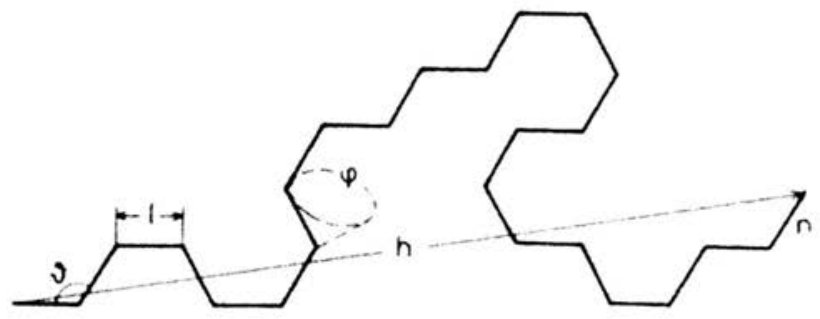

Figure 2. Model of freely-jointed polymer chain with rotation of neighbour bonds.

The consequences of the above features are entropy-controlled elasticity, wide range of molecular flexibility from thread to rigid rod, and no possibility of evaporation.

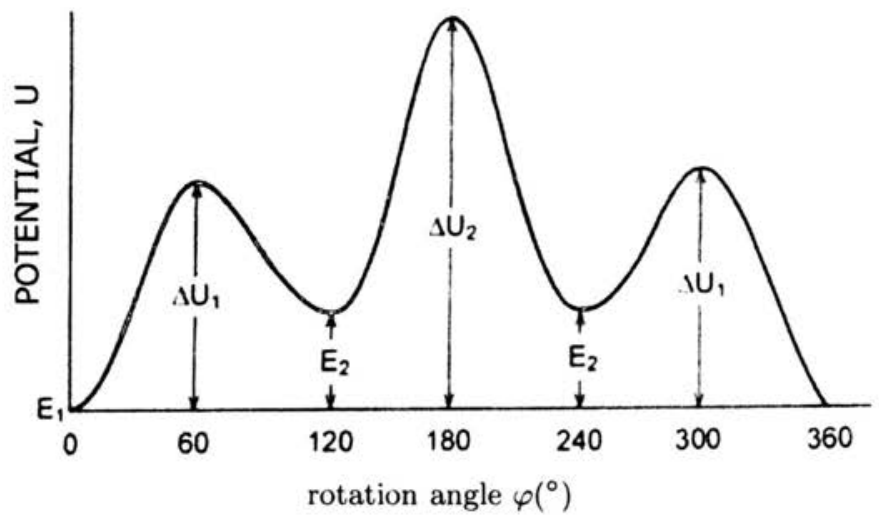

FIGURE 3. Rotational potential $U(\varphi)$.

Potential energy of intramolecular rotation is shown in Fig. 3. Transition from one (rotational) position to another one requires overcoming of a potential barrier $\Delta U$. The lower is this barrier, the easier is change of molecular shape and the macromolecule more resembles a flexible thread. In the state of equilibrium (maximum entropy), flexible molecular chain assumes shape of a coil (Fig. 4(a)). Low rotational barriers are characteristics of polymer chains composed of $\mathrm{C}-\mathrm{C}, \mathrm{Si}-\mathrm{Si}, \mathrm{C}-\mathrm{O}$ and $\mathrm{O}-\mathrm{O}$ bonds. On the other hand, rotational barriers for chains composed of aromatic rings (polyphenylenes) or double $\mathrm{C}=\mathrm{C}$ bonds (polyacetylene) are much higher. The equilibrium state of such molecules resembles rigid rod (Fig. $4(\mathrm{c})$ ). Intermediate rotational barriers give rise to semi-flexible molecules (Fig. 4(b)). Relations between the rotational barriers, degree of flexibility and statistical distribution of molecular conformations have been analyzed theoretically [2]-[5] and characteri- 
zed experimentally using infra-red or Raman spectroscopy. Table 1 presents potential barriers for rotation of various chemical bonds.

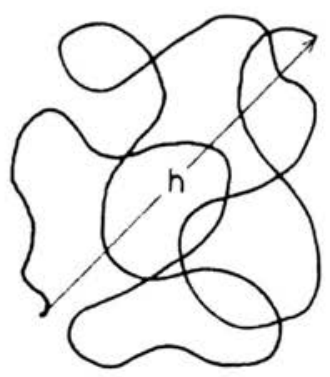

(a)

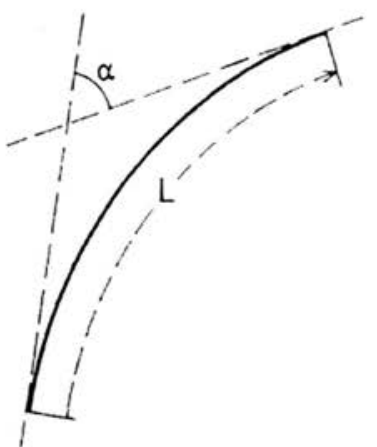

(b)

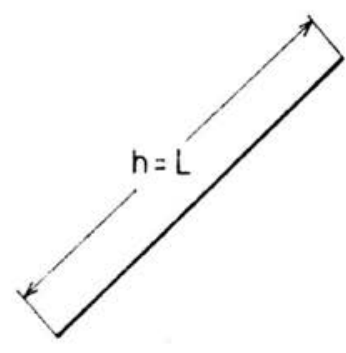

(c)

FIGURE 4. Equilibrium shapes of macromolecules with different degree of rigidity (a) flexible coil, (b) semi-rigid molecule, (c) ideally rigid rod.

TABLE 1. Potential barriers for molecular rotation, after [1].

\begin{tabular}{|c|c|c|}
\hline bond & molecule & $\Delta U, \mathrm{kcal} / \mathrm{mole}$ \\
\hline $\mathrm{C}-\mathrm{C}$ & $\mathrm{CH}_{3}-\mathrm{CH}_{3}$ & 2.75 \\
\hline $\mathrm{C}-\mathrm{C}$ & $\mathrm{CH}_{3}-\mathrm{CF}_{3}$ & $3.9-4.0$ \\
\hline $\mathrm{C}-\mathrm{C}$ & $\mathrm{CH}_{3}-\mathrm{CHCl}_{2}$ & 3.75 \\
\hline $\mathrm{C}-\mathrm{C}$ & $\mathrm{CH}_{3}-\mathrm{CH}_{2} \mathrm{Cl}$ & $3.4-4.47$ \\
\hline $\mathrm{C}-\mathrm{C}$ & $\mathrm{Cl}_{2} \mathrm{CH}-\mathrm{CHCl}_{2}$ & 4.5 \\
\hline $\mathrm{C}-\mathrm{C}$ & $\mathrm{H}_{5} \mathrm{C}_{6}-\mathrm{C}_{6} \mathrm{H}_{5}$ & 9.0 \\
\hline $\mathrm{C}-\mathrm{O}$ & $\mathrm{CH}_{3}-\mathrm{OH}$ & $1.07-1.09$ \\
\hline $\mathrm{C}-\mathrm{S}$ & $\mathrm{CH}_{3}-\mathrm{SH}$ & $0.7-1.06$ \\
\hline $\mathrm{C}-\mathrm{N}$ & $\mathrm{CH}_{3}-\mathrm{NH}_{2}$ & $1.90-1.97$ \\
\hline $\mathrm{C}-\mathrm{Si}$ & $\mathrm{CH}_{3}-\mathrm{SiH}_{3}$ & $1.5-1.7$ \\
\hline $\mathrm{C}=\mathrm{C}$ & $\mathrm{CH}_{2}=\mathrm{CH}_{2}$ & 39.3 \\
\hline $\mathrm{C}=\mathrm{C}$ & $\mathrm{CH}_{2}=\mathrm{C}=\mathrm{CH}_{2}$ & 27.2 \\
\hline $\mathrm{O}-\mathrm{O}$ & $\mathrm{HO}-\mathrm{OH}$ & 0.32 \\
\hline $\mathrm{O}-\mathrm{N}$ & $\mathrm{HO}-\mathrm{NO}_{2}$ & $10.0-14.2$ \\
\hline $\mathrm{N}-\mathrm{N}$ & $\mathrm{O}_{2} \mathrm{~N}-\mathrm{NO}_{2}$ & 0.6 \\
\hline $\mathrm{N}-\mathrm{N}$ & $\mathrm{H}_{3} \mathrm{~N}-\mathrm{NH}_{3}$ & 3.5 \\
\hline $\mathrm{Si}-\mathrm{Si}$ & $\mathrm{H}_{3} \mathrm{Si}-\mathrm{SiH}_{3}$ & $1.0-1.2$ \\
\hline
\end{tabular}




\subsubsection{Crystal structure $(0.1-100 \mathrm{~nm})$. Crystal lattice structure} (0.1-10 nm), including interplanar distances, and dimensions of crystallites $(1-100 \mathrm{~nm})$ is a characteristic similar to simple molecular crystals. One can ask, how is it possible that long macromolecular chains (with extended length reaching $10^{3} \mathrm{~nm}$ or more) fit into crystal lattice with interplanar distances of the order of $0.1-1 \mathrm{~nm}$ ? In the early studies of polymer structure Gerngross and Hermann [6], and later Kratky and Mark [7] introduced a model (Fig. 5(a)) in which only part of a long polymer chain is built into the crystal, other segments being a part of the amorphous phase. Each crystal is composed of a bundle of segments originating from many different macromolecular chains, and each macromolecule can participate in many different crystals and amorphous regions. This fringed micelle model seems to explain crystallization of polymers from melts or concentrated solutions.

Crystallization of short and rigid chains (especially under pressure) may give rise to extended-chain crystals composed of entire molecules (Fig. 5(b)).

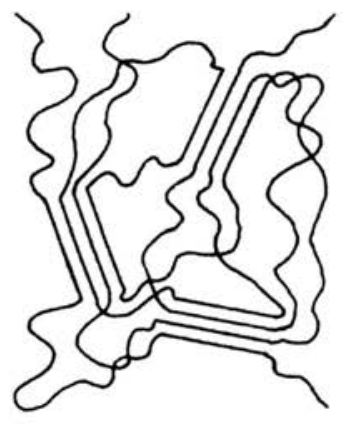

(a)

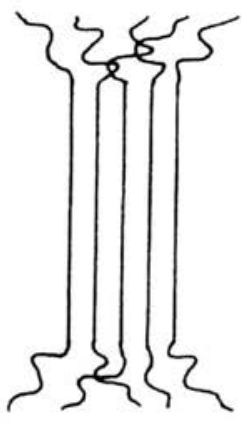

(b)

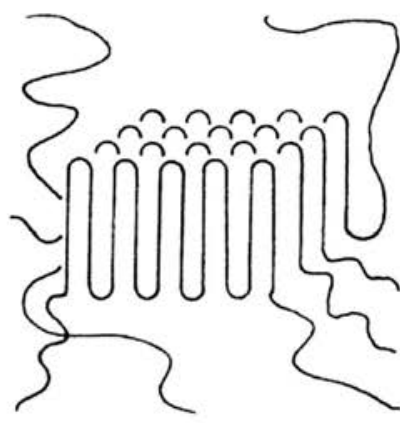

(c)

Figure 5. Molecular models of crystalline polymers (a) many-chain (fringed micelle) crystal, (b) extended-chain crystal, (c) intramolecular, folded-chain crystal.

Discovery of polymeric single crystals obtained from crystallization in very dilute solutions, offered another challenge to polymer scientists. Single crystals of polyethylene can be observed under electron microscope (Fig. 6). They have well defined pyramidal shape, and thickness of the order of $10 \mathrm{~nm}$ smaller by many orders of magnitude than length of an extended macromrolecule. An ingenious explanation offered by Keller [8], Price [9] and Peterlin [10] was based on the assumption that each crystal consists of many regularly folded chains (Fig. 5(c)). This existence of folded-chain crystals has been proved by direct experimental evidence.

Specific for polymers structure is degree of crystallinity - fraction(s) of the crystalline phase(s). Metals, alloys, ceramics, and many low-molecular 


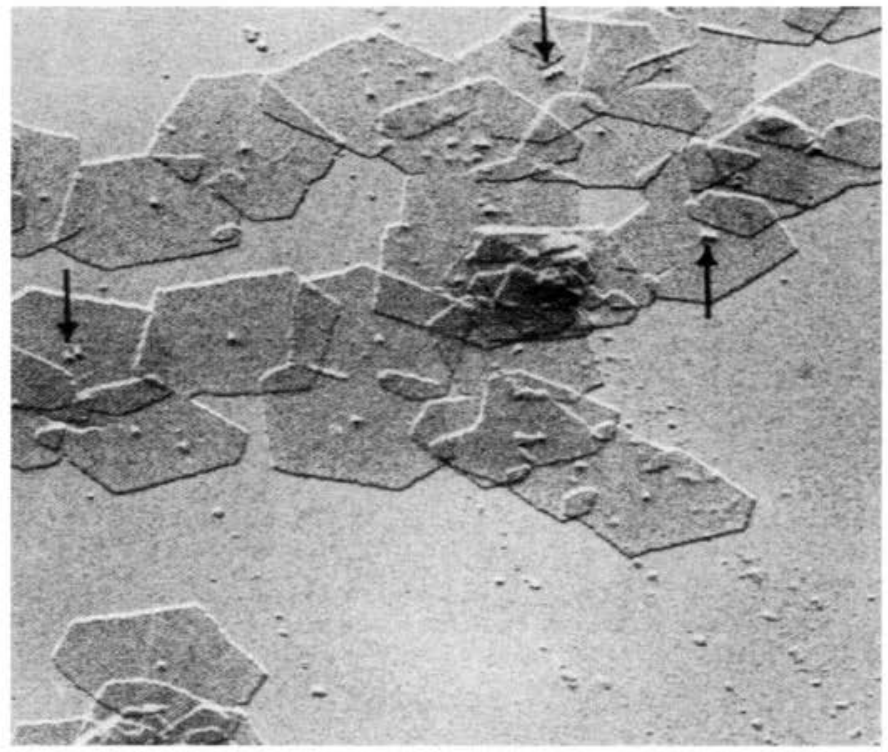

FIGURE 6. Electronomicrograph of polyethylene single crystal, crystallized from a dilute solution.

substances when cooled down below melting temperature crystallize fast and usually reach the state of equilibrium with complete transformation (degree of crystallinity $=1$ ). Crystallization of polymers is slow, and usually incomplete because of long relaxation times and sterical constraints which exclude formation of ideal order. Consequently, the same polymer may be undercooled in an amorphous state or crystallized partially to different values of the degree of crystallinity. Some polymers are completely incapable of crystallization.

The degree of crystallinity in crystallizable polymers varies from zero to $30-90 \%$ dependently on crystallization conditions (e.g. thermal and mechanical history) and affects mechanical, thermal, and optical properties. An important structural characteristic of polymers is molecular orientation. Deformation of long polymer chains, orientation distribution of chain segments and orientation of crystals strongly affect anisotropy of mechanical, electrical, sorptional and optical properties.

Experimental techniques used for characterization of crystal structure include X-Ray (electron, neutron) diffraction\&scattering, calorimetry, light scattering\&depolarization, optical birefringence and densitometry.

Wide-angle X-Ray Diffraction (WAXD) and Small-Angle X-Ray Scattering (SAXS) provide direct information about crystal lattice, degree of crystalline phase(s), crystal orientation, size and shapes of crystals. Typical WAXD patterns are shown in Fig. 7. It is evident that amorphous "halo" in 


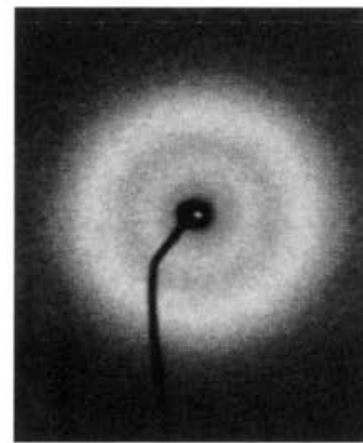

(a)

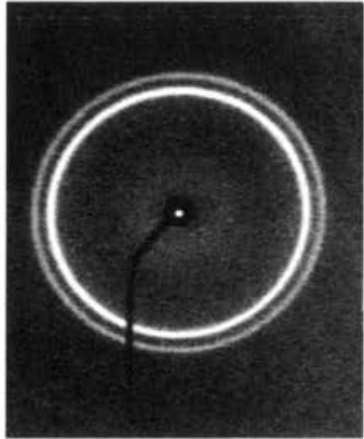

(b)

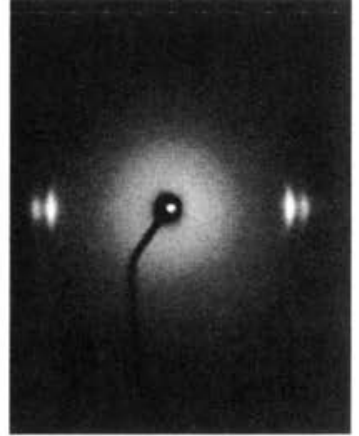

(c)

Figure 7. Wide-angle X-Ray diffraction patterns of polymers (a) amorphous, unoriented polyethylene terephthalate, (b) crystalline unoriented polyethylene, (c) oriented crystalline polyethylene.

amorphous polymer (Fig. 7(a)) changes on crystallization into a system of rings (Fig. 7(b)). Orientation of crystals (e.g. by deformation of the sample) makes crystalline rings to split into arcs (Fig. 7(c)). Position, width and intensity distribution in crystalline rings and arcs make basis for qualitative and quantitative analysis of the crystalline texture.

1.2.3. Supramolecular structure $(\mathbf{1 0}-\mathbf{1 0 6} \mathrm{nm})$. Large morphological forms in polymers include spherulites - radially or tangentially ordered aggregates composed of small crystals, fibrils, crystal plates, shish-kebab structures, row structures, and others. Examples of spherulites are shown in Fig. 8. Supramolecular (morphological) structure is experimentally investigated by polarizing microscopy, electron microscopy, low-angle light scattering. Spherulitic structue strongly affects optical properties of polymers. To increase transparency of (usually spherolitic) polyethylene films, crystal nucleating agent is added to the melt. This eliminates few, large, strongly light scattering spherulites and replaces them with many small crystals with suboptical sizes. Coarse-spherulitic structure on polymer surface was found to affect also frictional properties.

\subsection{Chemical bonds and interaction energies}

Table 2 presents energies of various types of chemical bonds. Energy of a single bond (ionic, metallic, hetero- or homeopolar) is high compared to intermolecular (van der Waals) interactions. However, single atom or small molecule interacts with a few of its neighbours, and interaction energy per molecule does not exceed energy of several (4-8) single bonds, i.e. 

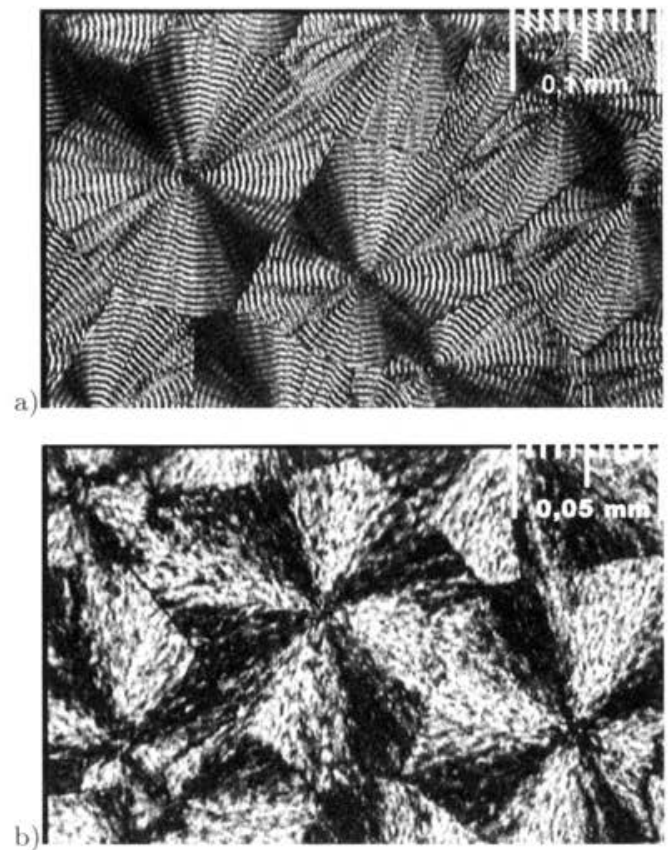

Figure 8. Polyethylene adipate spherulites under polarizing microscope a) ring spherulite, b) radial spherulite

TABLE 2. Bonding energies, after [1].

\begin{tabular}{|c|c|c|}
\hline bond type & example & energy, kcal/mole \\
\hline ionic & $\mathrm{NaCl}$ & 182 \\
\hline heteropolar & $\mathrm{ZnO}$ & 972 \\
\hline & $\mathrm{CaF}_{2}$ & 618 \\
\hline homeopolar & $\mathrm{C}-\mathrm{C}$ & 80 \\
\hline & $\mathrm{C} \equiv \mathrm{C}$ & 142 \\
\hline metallic & $\mathrm{Li}$ & 36.2 \\
\hline & $\mathrm{Cu}$ & 81 \\
\hline hydrogen bonds & $\mathrm{OH} \ldots \mathrm{O}$ & $5.9-10.15$ \\
\hline & $\mathrm{CH} \ldots \mathrm{N}$ & $6.1-7.8$ \\
\hline & $\mathrm{NH} \ldots \mathrm{F}$ & 6.0 \\
\hline van der Waals bonds & & \\
\hline plyethylene & $\mathrm{CH}_{2}, \mathrm{CH}_{2}$ & 1.0 \\
\hline polystyrene & $\mathrm{CH}_{2}, \mathrm{C}_{6} \mathrm{H}_{5}$ & 4.0 \\
\hline polyamide & $\mathrm{CH}_{2}, \mathrm{CONH}$ & 5.8 \\
\hline
\end{tabular}


$100-500 \mathrm{kcal} / \mathrm{mole}$. On the other hand, polymer chain composed of thousands of units, each unit interacting with energy of the order of 1-4 kcal $/ \mathrm{mole}$ experiences global interaction energy of the order of $10^{4}-10^{5} \mathrm{kcal} / \mathrm{mole}$. This excludes the possibility of evaporation because molecular destruction (dissociation of a single bond, however strong) requires only $50-100 \mathrm{kcal} / \mathrm{mole}$.

\subsection{Applications of polymer materials}

Versatility of polymer structure and properties enable wide spectrum of their applications. There are two basic groups of polymers.

Traditional polymers produced in the mass scale

- construction materials,

- textile and industrial fibers,

- packaging materials,

- non-woven materials,

- rubbers.

Special polymers manufactured in relatively small quantities

- high-modulus \& high-strength fibers for reinforcement of composites,

- piezoelectric polymers,

- conducting polymers,

- photo-sensitive polymers for lithography,

- polymer diodes, sensors and detectors,

- polymer electrolites for lithium batteries,

- polarization filters,

- polymers for medicine \& pharmacy (inteligent drugs, prostheses, etc.). Versatility of polymer structure, enables development of materials with desired properties and characteristics. Designing and synthesizing new polymer materials is a rapidly growing part of materials engineering.

\section{Rubbers. Topological structure and elasticity of polymer networks}

Statistical properties of polymer chains described in the preceding section, can be described quantitatively with simplified molecular models. Commonly used model is freely-jointed chain composed of $N$ rigid segments of length 
$a$ connected with $N-1$ joints (Fig. 2). Each segment can rotate freely with respect to its neighbours.

Random walk approach yields average end-to-end distance $(h)$ in the absence of stress in the form:

$$
\left\langle h^{2}\right\rangle=N a^{2} .
$$

Kuhn \& Grün [11] derived configuration distribution function

$$
W(h)=\text { const. } \exp \left[-\frac{1}{a} \int_{0}^{\mathbf{h}} L^{*}\left(\frac{x}{N a}\right) d x\right]
$$

where $L^{*}(x)$ is inverse Langevin function

$$
L^{*}(x)=3 x+\frac{9}{5} x^{3}+\frac{297}{175} x^{5}+\ldots
$$

Configurational entropy of the chain (treated as a statistical system) is obtained from $W(h)$ :

$$
S(h)=k \ln [W(h)]=S_{0}-\frac{k}{a} \int_{0}^{\mathbf{h}} L^{*}\left(\frac{x}{N a}\right) d x,
$$

and yields entropy-controlled free energy

$$
F(h)=U(h)-T S(h) \cong-T S(h)=\frac{k T}{a} \int_{0}^{\mathrm{h}} L^{*}\left(\frac{x}{N a}\right) d x,
$$

and elastic tension

$$
\begin{aligned}
\mathbf{f}(h)=\nabla F \cong-T \nabla S & =\frac{k T}{a h} L^{*}\left(\frac{h}{N a}\right) \mathbf{h} \\
& =\frac{3 k T \mathbf{h}}{N a^{2}}\left[1+\frac{3}{5}\left(\frac{h}{N a}\right)^{2}+\frac{99}{175}\left(\frac{h}{N a}\right)^{4}+\ldots\right] .
\end{aligned}
$$

Figure 9 shows force, $\mathbf{f}$, as a function of the degree of chain extension, $h / N a$. At small deformations, f linearly increases with $h$, at higher deformations turns up and tends to infinity at full extension $(h=N a)$.

The relations (2.1)-(2.6) explain the nature of rubber elasticity, a characteristic of flexible polymers. Unlike crystalline solids it is not internal energy, 


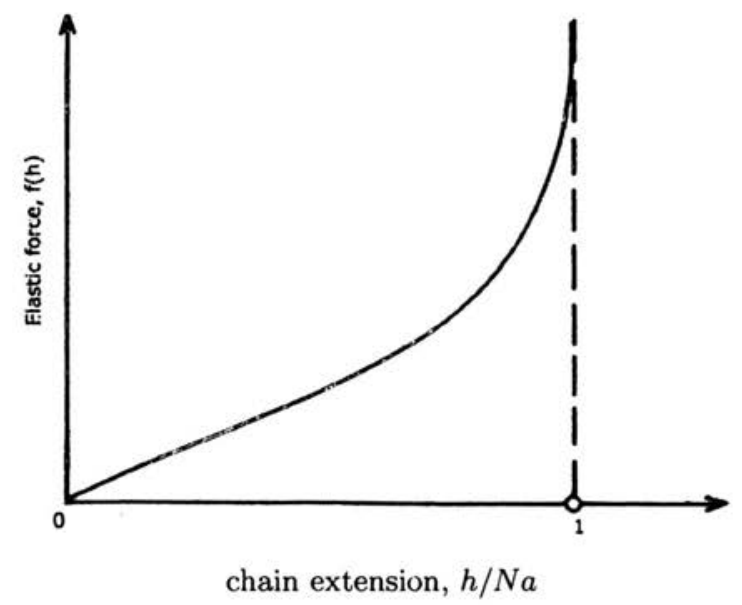

Figure 9. Elastic force in a polymer chain, vs. deformation, $h / N a$.

but entropy which gives rise to elastic behaviour. Elastic behaviour of polymers resembles gases. Compressibility of ideal gases, linear in the range of small volume changes, and non-linear in the van der Waals region) is also entropy controlled. Therefore, rubber elasticity is sometimes called gas-like behaviour.

Real polymers react to deformation also with some change of internal energy, $U(h)$. It is not explicit in the freely-jointed chain model, but energetical effects can be associated with transitions between rotational isomers (i.e. configurations corresponding to minima of potential energy $U(\varphi)$ (cf. Fig. 3). In the freely-jointed chain model, length of the statistical chain segmenta, and the number $N$ of such segments, can be treated as adjustible variables provided they satisfy two normalization conditions

$$
\begin{aligned}
N a & =L \\
N a^{2} & =\left\langle h^{2}\right\rangle
\end{aligned}
$$

Hydrodynamic (or contour) length $L$, of the chain and average square endto-end distance, $\left\langle h^{2}\right\rangle$ are molecular characteristics which can be measured experimentally. The higher is number $N$, of chain segments and the shorter is segment length $a$, the more flexible is the chain and the lower is its resistance to deformation. Internal energy effects can thus be included in the freely jointed chain model by reduction of the effective segment length.

Although physical source of entropy-controlled elasticity lies in the statistical behaviour of a single polymer chain, practical realization of rubberlike properties requires that many chains are connected into a network with macroscopic dimensions. 


\subsection{Formation of networks}

The process of joining polymer chains into larger structures is called crosslinking. Introduction of a small number of interchain connections ("crosslinks" or "junctions") produces molecular aggregates with statistically distributed sizes. When the density of crosslinks reaches some critical value (in the theory of Flory [12] - two crosslinks per primary chain) - a network is created. When crosslinking is performed in a solution, transition from a viscous fluid composed of single macromolecules and aggregates ("sol") into an elastic network swollen in the solvent ("gel") is called "gelation".

There are three main ways of crosslinking (Fig. 10):

1. Linear chains with reactive groups distributed along the chain react with a two-functional crosslinking agent creating "bridges" between two chains. The process called vulcanization leads to a tetrafunctional network. An example is provided by vulcanization of natural rubber with sulphur -SS- bridges.

2. $n$-functional (i.e. one carrying in each molecule $n$ reactive groups) monomer is subjected to polymerization, ultimately yielding an $n$ functional network. Functionality of the monomer must be at least 3 .

3. A mixture of linear chains with reactive groups on their ends is subjected to reaction with $n$-functional crosslinking agent $(n>2)$. The product is $n$-functional network.

Crosslinking can be result of chemical reactions between reactive groups of the uncrosslinked polymer molecules and groups of the crosslinking agent, reactive groups of identical or different polymer chains, as well as high-energy radiation (UV, gamma radiation). In the latter case, reactive groups or free radicals are created by high-energy radiation.

\subsection{Basic notions of the theory of networks}

Network junction (crosslink, node) - chemical, or physical bond between polymer chains. Crosslinks may consist of various kinds of homeopolar, ionic, hydrogen bonds or inclusion in a crystallite, or topological constraints (entanglements).

Functionality of a junction - number of polymer chains issuing from the junction.

Potential functionality of a junction - maximum number of polymer chains which can be attached to the junction; number of reactive groups at the junction. 
a)

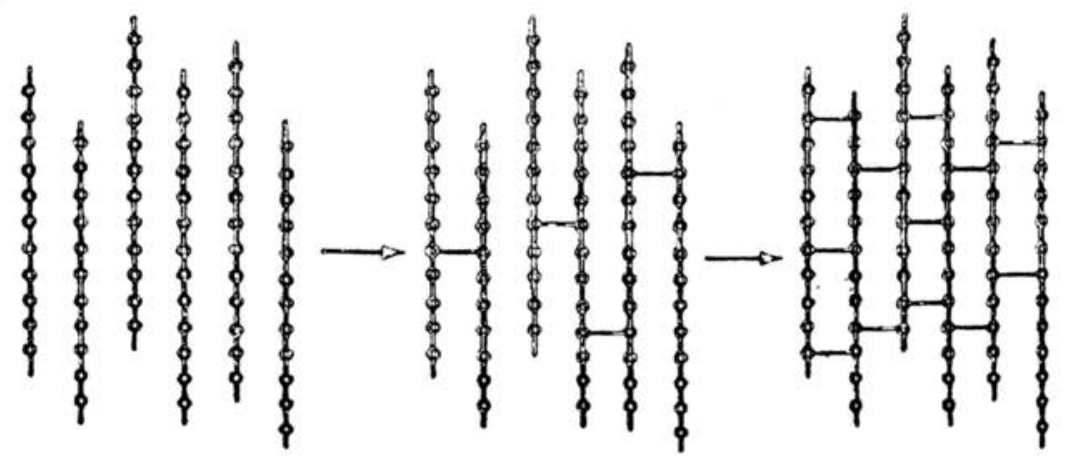

b)

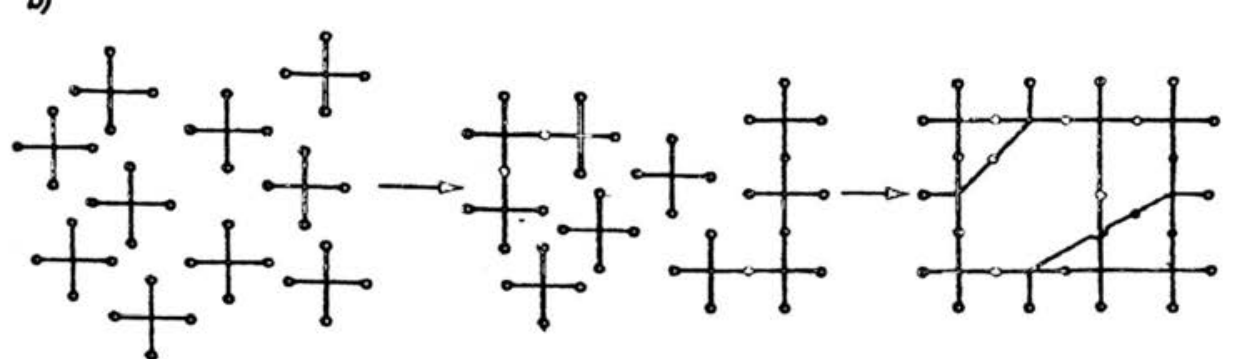

c)

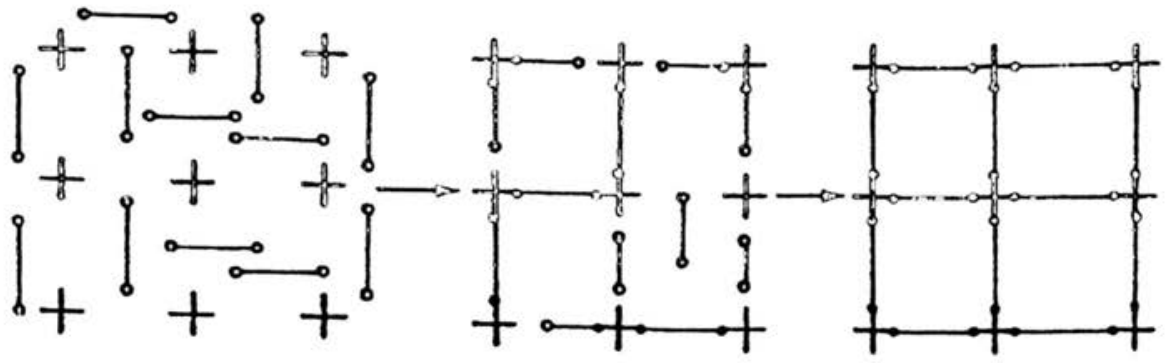

Figure 10. Various ways of crosslinking; a) vulcanization - formation of bridges along the contour of long linear chains, b) polymerization of a tetrafunctional monomer, c) attachment of reactive chain ends to a tetrafunctional crosslinking agent, after [21]. 
Real functionality of a junction - actual number of polymer chains attached to the junction; some potential functionalities can remain void.

Primary macromolecule - primary polymer chain before crosslinking.

Network chain - portion of a polymer macromolecule contained between two neighbour junctions. When crosslinking consists of connecting ends of primary chains to a crosslinking agent (Fig. 10(c)) network chains are identical with primary chains, vulcanization (Fig. 10(a)) yields network chains shorter than primary macromolecules.

Elastically effective junction - junction from which at least three different paths lead to sample surface.

Elastically effective network chain - network chain contained between two elastically effective junctions.

Chains contained in the soluble (sol) part of the system (chains connected to aggregates but not to the network), chains attached to the network with one end only, or with both ends to the same junction, do not transmit forces and are elastically ineffective. Ineffective chains contribute to dissipation of energy in rubbers.

\subsection{Equilibrium elasticity of networks}

Configurational free energy is a sum of energies of elastically effective network chains, $N_{\mathrm{ch}}$. It can be also expressed through the number of elastically effective junctions, $N_{\text {junct }}$,

$$
\begin{aligned}
F_{\text {net }}= & A \sum_{i=1}^{N_{\mathrm{ch}}} F_{\mathrm{ch}}\left(h_{i}\right)=A N_{\mathrm{ch}}\left\langle F_{\mathrm{ch}}(h)\right\rangle=N_{\text {junct }}\left\langle F_{\mathrm{ch}}(h)\right\rangle=\frac{3}{2} k T N N_{\text {junct }} \\
& \times\left[\left\langle\left(\frac{h}{N a}\right)^{2}\right\rangle+\frac{3}{10}\left\langle\left(\frac{h}{N a}\right)^{4}\right\rangle+\frac{99}{525}\left\langle\left(\frac{h}{N a}\right)^{6}\right\rangle+\ldots\right] .
\end{aligned}
$$

In Eq. (2.9) $N$ denotes number of statistical segments within the average elastically effective network chain. The "contraction factor" $A$, originally assumed as unity [12]

$$
A=1
$$

was later systematically derived by Walasek [13] from statistical physical considerations as

$$
A=\frac{N_{\text {junct }}}{N_{\text {ch }}}=\frac{2}{f}
$$

where $f$ is functionality of the junction. 
Elastic characteristics of rubber networks include shear modulus in the range $10-10^{3} \mathrm{kPa}$, and reversible elongation up to $1000 \%$. Note, that elastic free energy (and shear modulus) is proportional to the number of crosslinks, $N_{\text {junct }}$, and because of its entropy nature, the modulus is proportional to temperature, $T$.

\subsection{Ideal vs. real networks}

In an ideal $\mathrm{f}$-functional network each junction is connected to the same $(f)$ number of chains; no potential functionalities are left void. All network chains are connected with both ends to different junctions, and the system does not contain any sol fraction and elastically ineffective chains.

Real crosslinked systems, on the other hand, usually consist of an insoluble (gel) fraction and some soluble part (sol) which can be removed from the system via solvent extraction. Some chains attached to the gel fraction are elastically ineffective and do not contribute to modulus of elasticity. Individual junctions in the crosslinked system may carry different combinations of topological defects. Effect of free-end chains and loops on rubber elasticity have been recognized long ago [14]-[19]. More recently, a complete classification of topological structures in tri- and tetrafunctional networks has been developed [20]-[23].

\subsection{Structural elements and topologial classes of tetrafunctional network junctions}

We will consider all possible elements of topological structure of tetrafunctional $(f=4)$ networks (Fig. 11). 'F', 'S', 'V', 'D', ..., 'Q' denote 7 structural elements. The corresponding lower case characters ' $f$ ', 's', ' $v$ ', 'd', ..., 'q' represent junction occupation probabilities, or fractions of functionalities occupied by individual structural elements.

Monofunctional elements (each occupying one functionality of a junction) include free-end chains $\mathrm{F}$, attached to the crosslink with one end only, singlets $\mathrm{S}$, chain connecting two different junctions, and void (unsaturated) functionalities, V.

The two bifunctional elements are doublets, D - two chains in parallel connecting the same pair of junctions and loops L, originating from one chain connected with both its ends to the same junction.

Trifunctional (triplets, T) and tetrafunctional elements (quadruplets, Q) are analogical to doublets. Triplet is formed by three, and quadruplet by four parallel chains conncting a pair of junctions. 


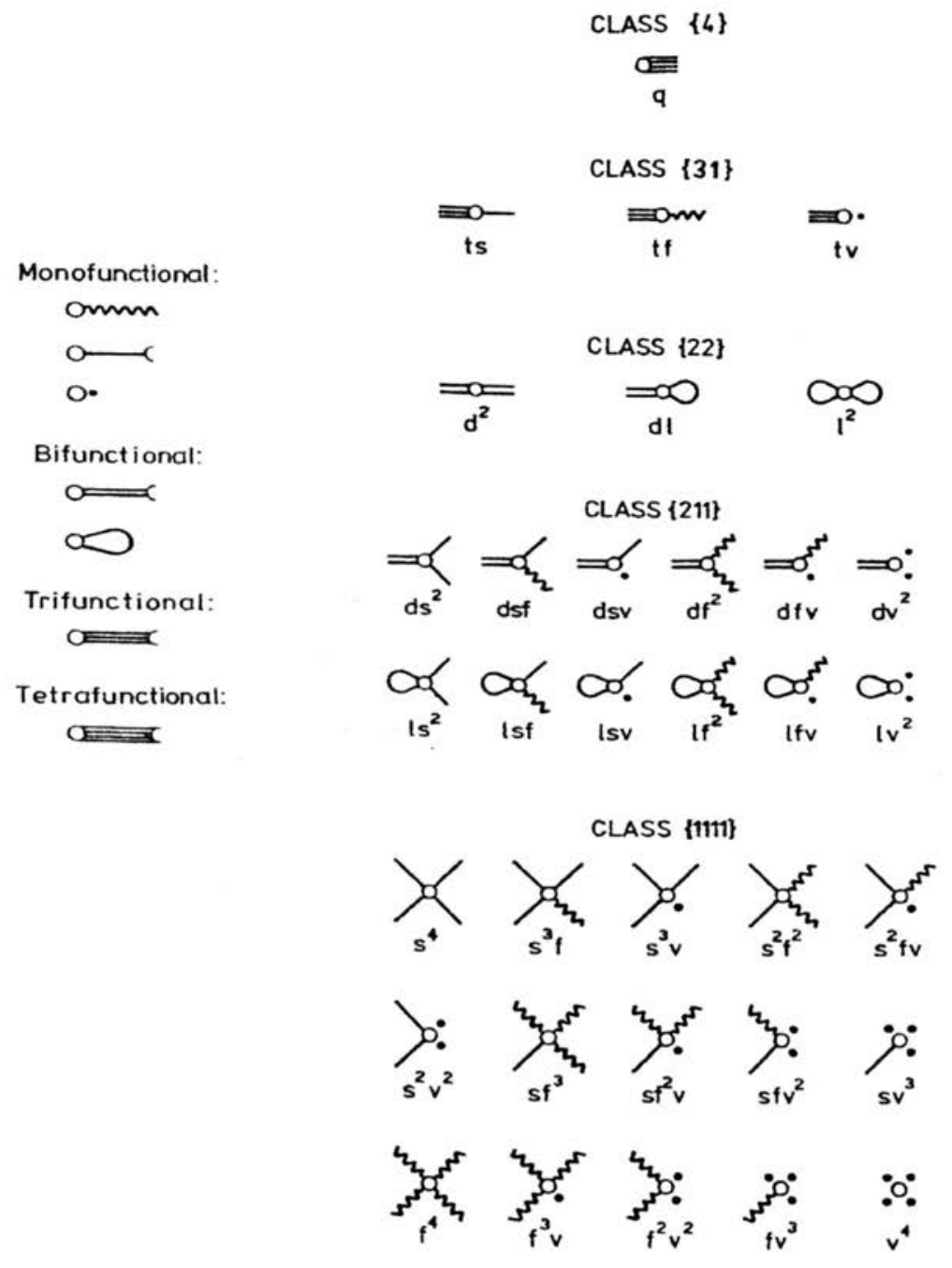

FIGURE 11. Left: structural elements; right: junction types, after [21].

In tetrafunctional crosslinked systems quadruplets can only appear in the sol as four-chain aggregates. Triplets and other elements may appear in the sol as well as in the gel fraction.

The fact that individual structural elements occupy different number (1, 2,3 , or 4 ) of junction functionalities, makes necessary distinction between four topological classes of tetrafunctional junctions. Class $\{4\}$ includes junction to which attached is one tetrafunctional element, class $\{3,1\}$ contains junctions with one trifunctional and one monofunctional element, class \{2,2\} - junctions with two bifunctional elements, class $\{2,1,1\}$ - with one bifunc- 
tional and two monofunctional, and class $\{1,1,1,1\}$ - with four monofunctional elements.

\subsection{Distribution of junctions in tetrafunctional networks}

Primary information about the structure is based on chemical and physico-chemical considerations and concerns the total number (concentration) of individual structural elements. Kinetics of crosslinking reactions, composition of the reacting mixture (constitution, concentration and molecular weight of polymer chains, concentration and structure of crosslinking agent) determine volume concentration of junctions, $N_{\text {junct }} / V$, fraction of void (unsaturated) functionalities and fraction of chains unattached to the gel.

2.6.1. Concentration of various structural elements. Important information about the fractions of functionalities occupied by free-end chains, $f=F / 4 N_{\text {junct }}$, unreacted crosslink functionalities $v$, singlets $s_{t}$, doublets $d$, loops, $l$, triplets $t$, and quadruplets $q$, is obtained from configurational statistics of polymer chains.Equilibrium probability distribution of end-toend distance, $W(h)$ is given by Eq. (2.2). For long enough chains, the distribution (2.2) can be reduced to Gaussian function

$$
\begin{aligned}
W(h) \cong & \left(\frac{3}{2 \pi N a^{2}}\right)^{3 / 2} \exp \left[-\frac{3 h^{2}}{2 N a^{2}}\right], \\
& \int_{0}^{\infty} 4 \pi h^{2} W(h) d h=1 .
\end{aligned}
$$

The probability that chain attached with one end to a crosslink will form a loop, a singlet, doublet, etc. depends on its length and flexibility characterized with the parameters $N$ and $a$. Consider a network chain attached with one end to a crosslink. The other end can either return to the same junction forming a loop, remain unattached (free-end chain) or attach to another junction, forming a singlet. Formation of a loop requires that the other chain finds itself in the small volume, $v_{0}$ at the distance $h=0$ from the first end. Probability of such an event is

$$
P(\text { loop })=v_{0} W(h=0)=\left(\frac{3}{2 \pi N a^{2}}\right)^{3 / 2} v_{0} .
$$

Now, let us consider formation of a singlet. To form a singlet, i.e. chain connecting two different junctions, the second end must find itself within 
a small volume $v_{0}$ in the vicinity of another crosslink. We assume that $N_{\text {junct }}$ crosslinks are randomly distributed in volume $V$. The probability $d P$ that the other chain end meets a crosslink within the infinitely thin spherical shell $\left(4 \pi h^{2} d h\right)$ is

$$
d P(\text { singlet })=\left(v_{0} \frac{N_{\text {junct }}}{V}\right) 4 \pi h^{2} W(h) d h,
$$

and, after integration over all end-to-end distances yields probability

$$
P(\text { singlet })=\left(v_{0} \frac{N_{\text {junct }}}{V}\right) 4 \pi \int_{0}^{\infty} W(h) h^{2} d h=\left(v_{0} \frac{N_{\text {junct }}}{V}\right) .
$$

The ratio of the above probabilites is identified with the ratio of the corresponding concentrations

$$
\frac{P(\text { loop })}{P(\text { singlet })}=\frac{l}{s}=\left(\frac{V}{N_{\text {junct }}}\right)\left(\frac{3}{2 \pi N a^{2}}\right)^{3 / 2}=Z \text {. }
$$

Similar considerations lead to the ratios

$$
\begin{gathered}
\frac{P(\text { doublet })}{P(\text { two singlets })}=\frac{d}{s^{2}}=\frac{\left(\frac{N_{\text {junct }}}{V}\right) \int_{0}^{\infty} W^{2}(h) h^{2} d h}{\left[\left(\frac{N_{\text {junct }}}{V}\right) \int_{0}^{\infty} W(h) h^{2} d h\right]^{2}} \\
=\left(\frac{V}{N_{\text {junct }}}\right)\left(\frac{3}{4 \pi N a^{2}}\right)^{3 / 2}=2^{-3 / 2} Z, \\
\frac{P(\text { triplet })}{P(\text { three singlets })}=\frac{t}{s^{3}}=\frac{\left(\frac{N_{\text {junct }}}{V}\right) \int_{0}^{\infty} W^{3}(h) h^{2} d h}{\left[\left(\frac{N_{\text {junct }}}{V}\right) \int_{0}^{\infty} W(h) h^{2} d h\right]^{3}} \\
=\left(\frac{V}{N_{\text {junct }}}\right)^{2}\left(\frac{\sqrt{3}}{2 \pi N a^{2}}\right)^{3}=3^{-3 / 2} Z^{2}, \\
\frac{P(\text { quadruplet })}{P(\text { four singlets })}=\frac{q}{s^{4}}=\frac{\left(\frac{N_{\text {junct }}}{V}\right) \int_{0}^{\infty} W^{3}(h) h^{2} d h}{\left[\left(\frac{N_{\text {junct }}}{V}\right) \int_{0}^{\infty} W(h) h^{2} d h\right]^{4}} \\
=\left(\frac{V}{N_{\text {junct }}}\right)^{3}\left(\frac{3 \sqrt[3]{2}}{\pi N a^{2}}\right)^{9 / 2}=\frac{1}{8} Z^{3} .
\end{gathered}
$$


Fractions of functionalities occupied by all structural elements are normalized

$$
f+s+v+l+d+t+q=1,
$$

and so are fractions of monofunctional

$$
f+s+v=\Sigma
$$

and bifunctional elements

$$
l+d=\Delta .
$$

2.6.2. Topological classes of tetrafunctional junctions. Class $\{4\}$ includes only one junction type $\{q\}$. Class $\{3,1\}$ comprises combinations of a triplet with three monofunctional elements $\{\mathrm{tf}\},\{\mathrm{ts}\},\{\mathrm{tv}\}$. To class $\{2,2\}$ belong junctions $\left\{\mathrm{l}^{2}\right\},\{\mathrm{ld}\}$, and $\left\{\mathrm{d}^{2}\right\}$. Class $\{2,1,1\}$ includes 12 types $\left\{\mathrm{lf}^{2}\right\}$, $\{l \mathrm{lfs}\},\{\mathrm{lfv}\},\left\{\mathrm{ls}^{2}\right\},\{\mathrm{lsv}\},\left\{\mathrm{lv}^{2}\right\},\left\{\mathrm{df}^{2}\right\},\{\mathrm{dfs}\},\{\mathrm{dfv}\},\left\{\mathrm{ds}^{2}\right\},\{\mathrm{dsv}\},\left\{\mathrm{dv}^{2}\right\}$, and class $\{1,1,1,1\}-15$ combinations of monofunctional elements $\left\{\mathrm{f}^{4}\right\},\left\{\mathrm{f}^{3} \mathrm{~s}\right\}$, $\left\{\mathrm{f}^{3} \mathrm{v}\right\},\left\{\mathrm{f}^{2} \mathrm{~s}^{2}\right\},\left\{\mathrm{f}^{2} \mathrm{sv}\right\},\left\{\mathrm{f}^{2} \mathrm{v}^{2}\right\},\left\{\mathrm{fs}^{3}\right\},\left\{\mathrm{fs}^{2} \mathrm{v}\right\},\left\{\mathrm{fs}^{2}\right\},\left\{\mathrm{fv}^{3}\right\},\left\{\mathrm{s}^{4}\right\},\left\{\mathrm{s}^{3} \mathrm{v}\right\},\left\{\mathrm{s}^{2} \mathrm{v}^{2}\right\}$, $\left\{\mathrm{sv}^{3}\right\},\left\{\mathrm{v}^{4}\right\}$.

All classes and configurations of junctions possible in tetrafunctional crosslinked systems are shown in Fig. 11.

Calculation of the fractions of individual junction types $n_{1}, \ldots, n_{34}$ is a two-step procedure. First calculated is probability of the topological class to which the junction belongs; next - probability of specific configuration within the class. The fraction of each junction type is:

$n_{k}=$ class probability $\times$ conditional probability of specific $k$-configuration .

Class probabilities read [20]-[23]:

$$
\begin{aligned}
P_{\text {class }}\{4\} & =q, \\
P_{\text {class }}\{31\} & =\frac{4 t}{3}, \\
P_{\text {class }}\{22\} & =\frac{\Delta^{2}}{\Delta+\Sigma-t / 3}, \\
P_{\text {class }}\{211\} & =\frac{2 \Delta(\Sigma-t / 3)}{\Delta+\Sigma-t / 3}, \\
P_{\text {class }}\{1111\} & =\frac{(\Sigma-t / 3)^{2}}{\Delta+\Sigma-t / 3},
\end{aligned}
$$

with normalization

$$
P_{\text {class }}\{4\}+P_{\text {class }}\{31\}+P_{\text {class }}\{22\}+P_{\text {class }}\{211\}+P_{\text {class }}\{1111\}=1 \text {. }
$$


2.6.3. Examples. The following formulae illustrate the two-step calculation procedure:

$$
\begin{aligned}
n_{k}(q) & =P_{\text {class }}\{4\} \times P_{\text {cond }}[q ;\{4\}]=q \times 1=q, \\
n_{k}(t s)= & P_{\text {class }}\{31\} \times P_{\text {cond }}[s ;\{31\}]=\frac{4 t}{3} \times \frac{s}{\Sigma}=\frac{4 t s}{3 \Sigma}, \\
n_{k}\left(d^{2}\right)= & P_{\text {class }}\{22\} \times P_{\text {cond }}[d d ;\{22\}] \\
= & \frac{\Delta^{2}}{\Delta+\Sigma-t / 3} \times \frac{d^{2}}{\Delta^{2}}=\frac{d^{2}}{\Delta+\Sigma-t / 3}, \\
n_{k}(d l)= & P_{\text {class }}\{22\} \times P_{\text {cond }}[d l ;\{22\}] \\
= & \frac{\Delta^{2}}{\Delta+\Sigma-t / 3} \times \frac{2 d l}{\Delta^{2}}=\frac{2 d l}{\Delta+\Sigma-t / 3}, \\
n_{k}(d s f)= & P_{\text {class }}\{211\} \times P_{\text {cond }}[d s f ;\{211\}] \\
= & \frac{2 \Delta(\Sigma-t / 3)}{\Delta+\Sigma-t / 3} \times \frac{2 d s f}{\Delta \Sigma^{2}}=\frac{4 d s f(\Sigma-t / 3)}{(\Delta+\Sigma-t / 3) \Sigma^{2}}, \\
n_{k}\left(s^{2} v f\right)= & P_{\text {class }}\{1111\} \times P_{\text {cond }}\left[s^{2} v f ;\{1111\}\right] \\
& =\frac{(\Sigma-t / 3)^{2}}{\Delta+\Sigma-t / 3} \times \frac{12 s^{2} v f}{\Sigma^{4}}=\frac{12 s^{2} v f(\Sigma-t / 3)^{2}}{(\Delta+\Sigma-t / 3) \Sigma^{4}} .
\end{aligned}
$$

The general form of a constrained polynomial distribution reads:

$$
n_{k}=C_{k}(\alpha, \beta, \gamma, \delta, \varepsilon, \eta, \lambda) \times f^{\alpha} s^{b} v^{\gamma} l^{\delta} d^{\varepsilon} t^{\eta} q^{\lambda} .
$$

Coefficients $C_{k}$ for all junction types have been described in [22, 23].

\subsection{Junction distribution and properties of networks}

Concentration of crosslinks, $N_{\text {junct }} / V$ and configuration of various structural elements control physical properties of rubbers and rubberlike materials. On the basis of junction distribution $n_{k}$ important properties of a crosslinked system can be predicted and modified. Structure-controlled properties include concentration of elastically effective junctions, fractions of chains attached and unattached to the network (soluble sol, insoluble gel fractions), critical concentration of crosslinks for the $s o l \rightarrow g e l$ transition ( $g e l$ point), modulus of elasticity, energy dissipation characteristics and others. All the properties, related to molecular characteristics of the system, can be predicted, modified and controlled. 


\subsection{Permanent vs. temporary, localized vs. entanglement networks}

In addition to ideal networks with permanent chemical bonds as crosslinks, real polymers may include different kinds of bonds between polymer chains which in some conditions behave like crosslinks, in other contribute to energy dissipation. First, one should distinguish between permanent and temporary (dissociable) junctions. As evident in Table 2 in Sec. 1 above, chemical bonds do not exhibit infinite strength. Application of high enough energy (thermal or mechanical) leads to dissociation of the bond and separation of molecular fragments. On the other hand, when subcritical energy (say, tension) is applied, forces from one chain are transmitted to the other one via crosslink without any relative displacement (Fig. 12(top)). Such junctions are localized. Breaking (dissociation) of junctions occurs when critical energy (force) is applied. Industrial rubber networks considered as permanent contain chemical crosslinks with high (but not infinite!) dissociation energy, say $80 \mathrm{kcal} / \mathrm{mole}$. On the other hand, concentrated polymer solutions may create labile networks with very weak bonds - dissociation energy based on hydrogen bonds with energy not exceeding $3-5 \mathrm{kcal} / \mathrm{mole}$. Such networks are thermally reversible. Crosslinks are destroyed by heating and recreated on cooling. A typical example is aqueous gelatin solutions. Short-time response of temporary networks is elastic while steady-state flow contributes to energy dissipation and provides one of the existing rheological
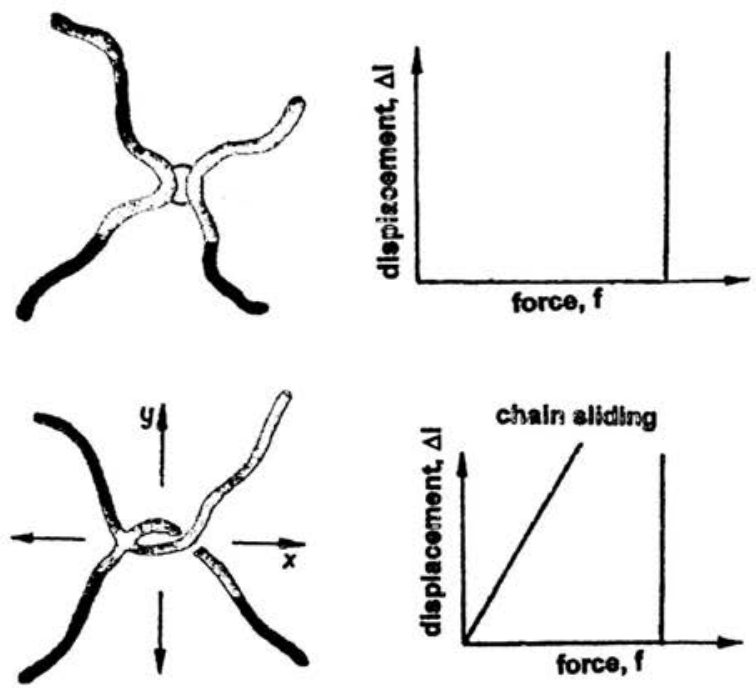

Figure 12. Behaviour of a localized (top) and entanglement junction (bottom). 
models of concentrated polymer solutions $[24,25]$. The role of localized but dissociable junctions can be played by crystals providing "bridges" between network chains and affecting modulus of elasticity [26]. Crystallization role is dual. On one hand, stress in a deformed network induces crystallization On the other one, crystallinity increases the number of elastically effective junctions and makes rubber stiffer. Crystallization is one of the sources of non-linear elastic behaviour.

Another type of network junctions is topological constraint called chain entanglement [27, 31]. Dense systems of long, flexible chains contain many obstacles to relative displacement. Some of such obstacles are permanent (e.g. interpenetrating rings, entanglements entrapped between chemical crosslinks) and contribute to equilibrium modulus of elasticity. Those with free ends disengage in the process of deformation and affect viscoelastic response. Figure 12 (bottom) presents entanglement junction and its response to applied force $\mathbf{f}$. Note, that chains participating in entanglement are not localized. In addition to transmission of elastic forces, chains slide one past another, and the sliding friction contributes to energy dissipation. When high enough force is applied, the chains break.

\subsection{Viscoelastic properties of polymer networks}

Although equilibrium elasticity, linear in the range of moderate deformations is a typical feature of single polymer chains and ideal rubbers, real crosslinked systems, especially those containing topological defects, entanglements and dissociable junctions exhibit wide range of non-linear viscoelasticity. One of the main sources of non-linear rubber elasticity is statistical behaviour of a single chain at large deformations (see Fig. 12). At high deformations (forces) applied to a network, deformation of network chains is non-affine and network junctions rearrange in space, trying to minimize their free energy. The same can be said about entanglement junctions entrapped between permanent crosslinks [31]. Portions of network chains engaged in an entanglement slide one with respect to another, assuming new deformation-dependent positions. The latter effects reduce modulus compared to an "affine". On the other hand, deformartion-induced crystallization leads to an increase of the modulus. One of the source of viscous effects is all topological defects which make some network junctions elastically ineffective (loops, free-end chains). Displacement of such structures, chain sliding in entrapped entanglements as well as disengagement of free-end entanglements lead to dissipation of energy and deteriorate rubber properties [32]-[34]. 


\section{Crystal nucleation in polymers. Effects of external fields and internal structure}

Kinetics of structure formation plays important role in determining physical properties of metals, polymers and other materials. The classical model developed by Kolmogoroff [35], Avrami [36], Johnson and Mehl [37] and Evans [38] presents crystallization as a sequence of two kinetic processes: nucleation and growth and crystal growth in one, two, or three dimensions (Fig. 13). The concept of nucleation, applied to various phase transitions, consisting of formation of stable atomic or molecular clusters, capable of spontaneous growth to microscopic dimensions.

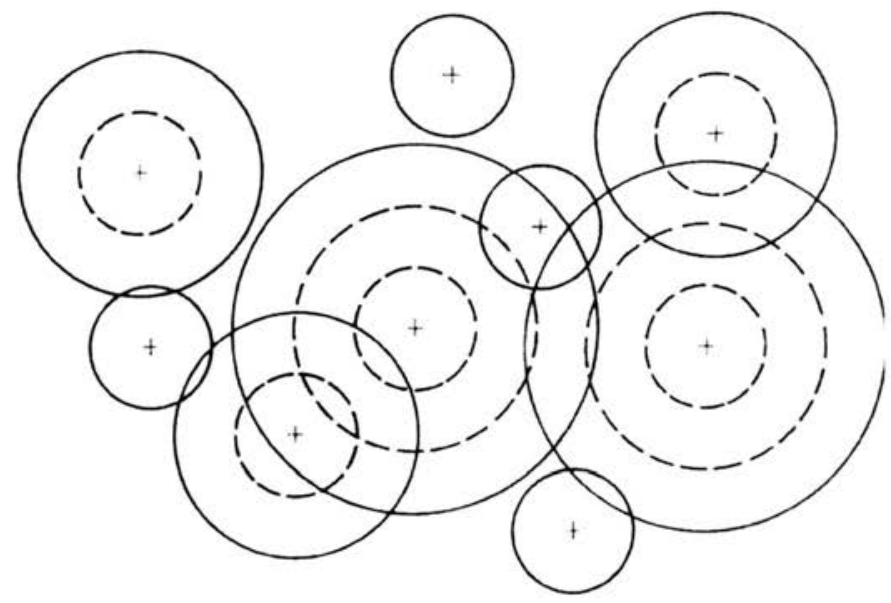

FIgURE 13. Crystallization as a random process of growing centers (nuclei).

Statistical theory of nucleation developed in the first half of XX century by Volmer and Weber [39], Becker and Döring [40], Frenkel [41] amd Turnbull [42], describes reasonably well simple phase transitions like condensation of gases, separation of liquids or crystallization of metals. Although widely used also to crystallization of polymer, it can not explain many effects of critical importance for development of polymer structure.We have developed a generalized theory of nucleation $[43,45]$ which predicts new effects and is free from many shortcomings of the classical model.

\subsection{Basic concepts of the classical theory}

The theory considers growth o clusters having structure of the target phase (say: crystalline) from kinetic elements (atoms, moleculas, molecular segments) of the mother phase (say: melt). The basic (the only one) chara- 
cteristic of a cluster is its volume $v$, or the number $g$ of combined kinetic elements:

$$
g=v / v_{0} .
$$

It is implied that clusters are produced from spherical atoms or molecules and neither shape or orientation of the aggregating units plays any role. Analyzed is distribution density, probability density in one-dimensional space of cluster sizes

$$
w(g, t)=\frac{1}{N_{0}} \frac{d N(g)}{d g} .
$$

Time-dependent distribution function $w(g, t)$ is found from the continuity equation (a variety of Focker-Planck, or Smoluchowski-Einstein equation)

$$
\frac{\partial w(g, t)}{\partial t}+\frac{\partial j(g, t)}{\partial g}=0
$$

where $j$ is one-dimensional flux of growth

$$
j(g)=-D_{\mathrm{gr}}\left[\frac{\partial w}{\partial g}+\frac{w}{k T} \frac{\partial \Delta F}{\partial g}\right],
$$

consisting of diffusional ('diffusion of growth' coefficient $D_{\mathrm{gr}}$ ) and potential components. Free energy of cluster formation

$$
\Delta F(g)=F_{g}-g F_{1}=\frac{g v_{0} \Delta h\left(T-T_{m}\right)}{T_{m}}+\left(g v_{0}\right)^{2 / 3} \sigma
$$

plays the role of driving force for cluster growth. $\Delta \mathrm{h}$ is heat of crystallization, and $\sigma$ - interface energy. Above critical transition temperature $T>T_{m}$ is positive and increases to infinity with cluster size (Fig. 14). Below $T_{m}$

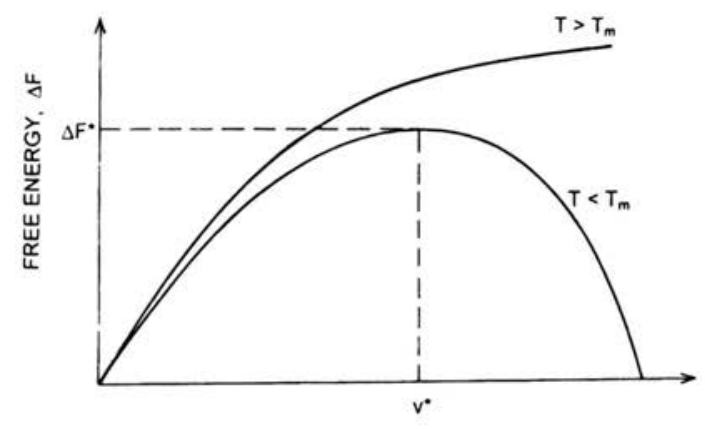

CLUSTER VOLUME, $v$

Figure 14. Free energy $\Delta F$ of an atomic (molecular) cluster as a function of volume $v^{*}$. Critical cluster size $v^{*}$ determines stable clusters (nuclei). 
(and this is the region where phase transition is thermodynamically possible) cluster free energy consists of two parts. Bulk component (proportional to cluster volume) is negative and tends to minus infinity with cluster size, $v$. On the other hand, energy of cluster surface (also edges, corners, etc.) is positive and increases with lower powers of volume $\left(v^{2 / 3}\right.$ for surface, $v^{1 / 3}$ for edges, etc.). The resulting $\Delta F(g)$ function exhibits a maximum at some critical cluster size, $g^{*}$ (Fig. 14),

$$
\begin{aligned}
& \frac{\partial \Delta F(g)}{\partial g}= 0 \Rightarrow g^{*}=\left[\frac{2 \sigma T_{m}}{3 \Delta h\left(T_{m}-T\right) v_{0}^{1 / 3}}\right]^{3}, \\
& \Delta F\left(g^{*}\right)=\Delta F^{*}=\Delta F_{\max } .
\end{aligned}
$$

Note, that subcritical clusters $\left(g<g^{*}\right)$ lying on the left (increasing) branch of the potential curve $\Delta F(g)$ are thermodynamically unstable: their growth requires an increase of energy. On the other hand, supercritical clusters $\left(g>g^{*}\right)$ may grow spontaneously because their growth is accompanied by reduction of energy. Supercritical (stable) clusters are called nuclei. Maximum free energy, $\Delta F^{*}$, provides potential barrier which small clusters must overcome to become stable nuclei.

The number of stable clusters ( $=$ nuclei) at the instant $t$ is

$$
\frac{N(t)}{N_{0}}=\int_{g^{*}}^{\infty} w(g ; t) d g,
$$

and nucleation rate

$$
\frac{\dot{N}(t)}{N_{0}}=\frac{d}{d t} \int_{g^{*}(t)}^{\infty} w(g, t) d g=\int_{g^{*}(t)}^{\infty} \frac{\partial w}{\partial t} d g-w\left(g^{*}\right) \frac{\partial g^{*}}{\partial t} .
$$

The first term in Eq. (3.8) describes thermal nucleation - growth of subcritical clusters to the critical size $g^{*}$. Combined with Eq. (3.3) thermal nucleation rate reduces to the flux of clusters over the potential barrier $\Delta F^{*}$. Thermal nucleation is the only mechanism of nucleation when crystallization conditions do not change in time and critical cluster size $g^{*}$, is a constant. However, in variable conditions (cooling, variable pressure, etc) $g^{*}$ is a function of time giving rise to another term in Eq. (3.8) - called by Hollomon et al. [46] athermal nucleation.

Athermal nucleation consists in "promotion" of subcritical clusters to the status of stable nuclei as a result of redefinion of critical size $g^{*}$. Redefinition 
is a natural consequence of the change of temperature, pressure or other state parameters. The rate of athermal nucleation is proportional to the rate of change of external conditions (say: cooling rate, $\dot{T}$ ) and does not require thermal activation or jumping over potential barriers:

$$
\frac{\dot{N}_{\text {ath }}}{N_{0}}=-w\left(g^{*}\right)\left[\frac{\partial g^{*}}{\partial T} \frac{d T}{d t}+\frac{\partial g^{*}}{\partial p} \frac{d p}{d t}+\ldots\right]
$$

The following two facts motivated our extension of the classical theory. First, polymer crystallization is very sensitive to molecular orientation. Crystallization from slightly oriented amorphous polymer leads to highly oriented crystals. Second, crystallization morphology, with anisotropic folded-chain crystal plates, cannot neglect shape effects. Neither orientation nor shape effects can be explained with one-dimensional theory.

\subsection{Basic concepts of the generalized theory}

The generalized theory was developed gradually, by introduction of various effects until a more systematic treatment was published in [44] and applications reviewed in [45]. Instead of one variable (size of the cluster $g$ ), $\mathrm{N}$-dimensional space of cluster configurations $\mathbf{V}$ is introduced. The configuration of the cluster is characterized with configurational vector, $\xi$, consisting of four groups of components

$$
\boldsymbol{\xi}=\{\mathbf{r}, \boldsymbol{\theta}, \mathbf{x}, \boldsymbol{\sigma}\} \in \mathbf{V}
$$

$\mathbf{r}$ is a group of $n$ coordinates characterizing cluster dimensions. For the model of a convex polyhedron (Fig. 15), $r_{i}(i=1,2, \ldots, n)$ are vectors normal to

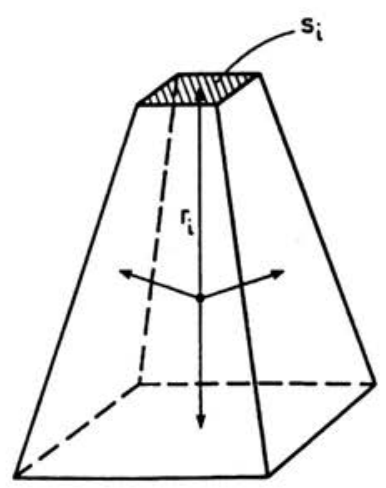

Figure 15. Convex polyhedral cluster. Radii normal to individual faces $r_{i}$, and surface areas $s_{i}$, indicated. 
individual crystal (cluster) faces. Directions of vectors

$$
\mathbf{r}=\left\{r_{1}, r_{2}, r_{3}, \ldots, r_{n}\right\}
$$

It should be mentioned that cluster shape effects were discussed also by other authors $[47,48]$. The next group of variables is cluster orientation $\boldsymbol{\theta}$ with respect to a fixed, external coordinate system. For this purpose three Euler angles are used

$$
\boldsymbol{\theta}=\{\vartheta, \varphi, \psi\} .
$$

Orientation is important when clusters and aggregating kinetic units are not spherical. Figure 16(a) illustrates schematically aggregation of spheres into a spherical cluster, and Fig. 16(b) - aggregation of rods into a rodlike cluster. The only requirement for spherical aggregation is that cluster and the element to be attached undergo collision or meet in a common volume. This condition determines cross-section for collisions in the positional space. For aggregation of asymmetric particles (Fig. 16(b)) the condition of collision is not sufficient. To make the attached element a component of the asymmetric (anisotropic) cluster - not only positions but also orientations of the reacting particles must be compatible. This imposes an additional condition of reaction cross-section in the rotational space. Simple statistical mechanical model of such cross-sections has been presented in $[49,50]$. The requirement of consistent orientation seems to be a general condition of phase transitions and chemical reactions involving asymmetric molecules. The direct consequence is increased entropy of the transition (reaction) and depressed critical transition temperature $T_{m}$.

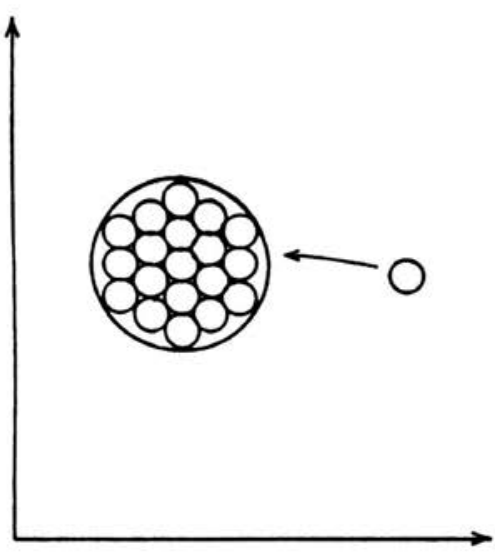

(a)

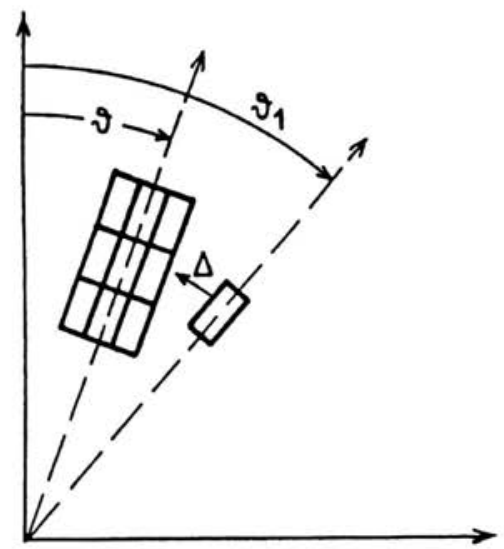

(b)

Figure 16. Aggregation of spherical (a) vs. cylindrical (b) particles. 
In polymers, the role of orientation is particularly important. It is not only that crystallizing polymer molecules (molecular segments) are asymmetric and orientable. Deformation, stress, external orienting fields etc. often produce in polymers state of orientation. Consider crystallization in an orienting field, $E_{\theta}$, with axis along the direction $\vartheta=0$. The field produces steady-state orientation distribution of chain segments in the mother phase. Free energy of aggregation includes an orientation-dependent entropy term which affects critical temperature for the transition $T_{m}=T_{m}\left(\theta ; E_{\theta}\right)$ and nucleation rates. This makes nucleation (and crystallization) selective: orientational entropy of segments oriented parallel to the field $(\vartheta=0)$ is small and their transition to the crystalline state is facilitated; aggregation of segments perpendicular to the field $(\vartheta=\pi / 2)$ is suppressed. The orientation-dependent transition temperature, $T_{m}(\vartheta)$ is shown in Fig. 17(bottom). Differentiation of the tran-
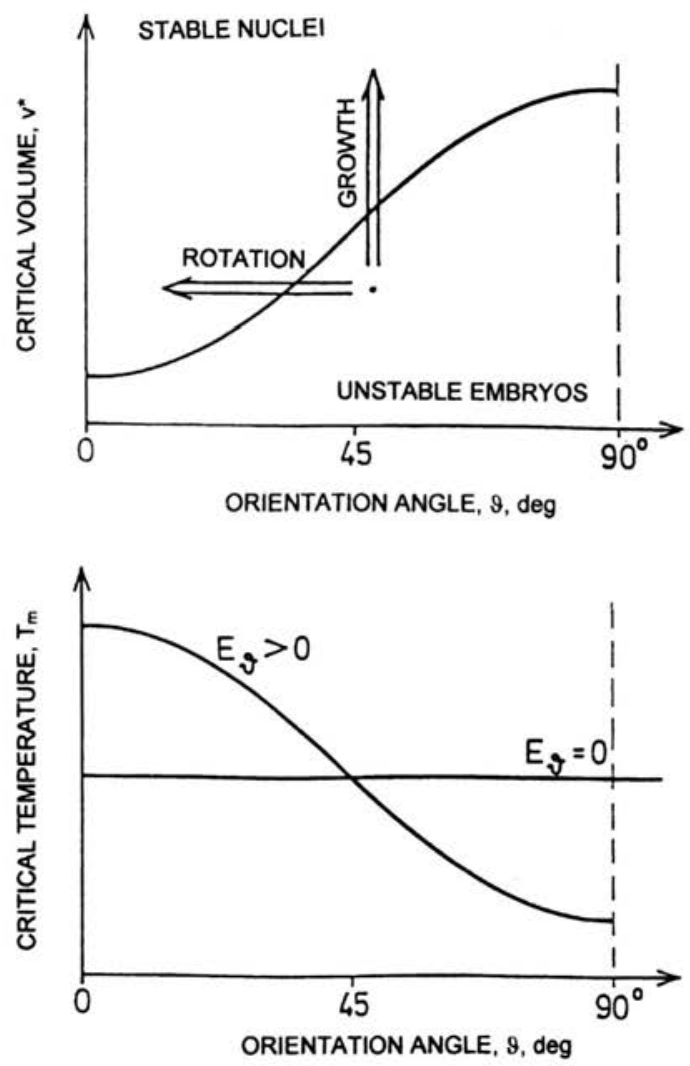

FigurE 17. Nucleation of asymmetric particles in an orienting field, $E_{\vartheta}$ top: critical volume $v^{*}$ vs. orientation $\vartheta$. bottom: critical transition temperature $T_{m}$ vs. orientation $\vartheta$, after [44]. 
sition temperature and selectivity of nucleation increases with intensity of the orienting field and disappears for $E_{\theta}=0$.

When different cluster orientations are admitted, it is only natural to introduce also their positions $\mathbf{x}$, with respect to an external coordinate system associated with a field with linear gradient, $E_{x}$.

$$
\mathbf{x}=\{x, y, z\}
$$

Gravitational field and electrostatic field acting on charged particles provide most natural examples. In such a field, free energy will be dependent on cluster position $\mathbf{x}$, and will lead to spatial distribution of transition conditions.

Last, not least, we introduce into our configurational vector $k$ parameters representing internal structure of the cluster - defects, distribution of cocomponents, etc.

$$
\boldsymbol{\sigma}=\left\{\sigma_{1}, \sigma_{2}, \ldots, \sigma_{\kappa}\right\}
$$

Their importance is justified by sensitivity of free energy to cluster structure.

Having defined $N$-dimensional $(N=6+n+k)$ configurational space, we analyze the appropriate distribution density

$$
w(\xi, t)=\frac{1}{N_{0}} \frac{d N}{d \xi}
$$

and the corresponding equation of continuity

$$
\frac{\partial w(\xi, t)}{\partial t}+\operatorname{div}_{\xi} \mathbf{j}(\xi, t)=0
$$

with flux

$$
\mathbf{j}(\xi, t)=-\mathbf{D}_{\xi}\left[\nabla_{\xi} w+\frac{w}{k T} \nabla_{\xi}(\Delta F)\right]
$$

Here $\operatorname{div}_{\xi}$ and $\nabla_{\xi}$ are differential operators in the configurational space [51] 
characterized with the metric tensor:

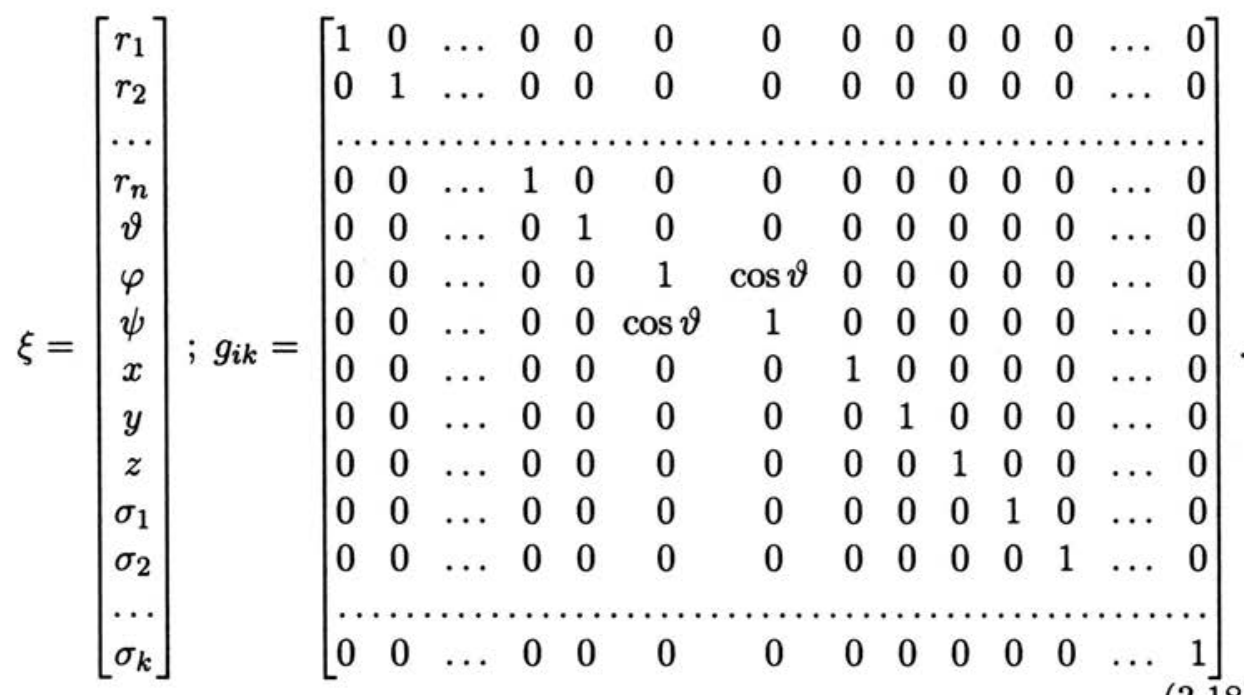

It is evident that the space is not Euclidean. Non-diagonal $g_{i k}$ components appear in the rotational region as associated with Euler angles.

The way in which the above model will be used for solving specific nucleation problems depends on free energy of cluster formation

$$
\Delta F(\boldsymbol{\xi})=\Delta F(\mathbf{r}, \boldsymbol{\theta}, \mathbf{x}, \boldsymbol{\sigma}) .
$$

Except for cluster dimensions $\mathbf{r}$ which are basic characteristics of a cluster, other components of the configurational vector $\boldsymbol{\xi}$, may appear, or not in the free energy. Whenever free energy is independent of a given component of $\boldsymbol{\xi}$, this component (orientation, position, internal structure) can be neglected and nucleation can be described in a more narrow space. The crucial role is played by external potential fields and sensitivity of free energy to internal structure.

Cluster orientation $\boldsymbol{\theta}$ has to be considered if (and only if) an external orienting field is active during nucleation or, if a nucleation proceeds in a pre-oriented system. In polymers, orientation relaxes slowly and even in the absence of active fields non-relaxed orientation distribution may lead to selective, orientation-induced effects.

The same can be said about cluster position, $\mathbf{x}$. In the presence of an active gravitational or electrostatic field, nucleation temperatures and rates are non-uniformly distributed in space, dependently on the distance from the poles. In non-uniform systems positional effects may be reduced by translational diffusion. 
Effect of internal structure on cluster free energy is universal. However, the appearance of parameters $\boldsymbol{\sigma}$ concerns only materials with high sensitivity to internal structure.

Critical conditions of cluster stability determine in the configurational space $V$, region of stability $V^{*}$, and separated from the rest of space by critical hypersurface $S^{*}$

$$
V^{*} \subset V ; \quad S^{*} \subset V .
$$

The condition of cluster stability is postulated in the form

$$
\frac{\partial \Delta F(\boldsymbol{\xi})}{\partial r_{i}} \leqslant 0 \text { for } i=1,2, \ldots, n \Rightarrow \boldsymbol{\xi} \in V^{*} .
$$

$N$-dimensional flux j, (Eq. (3.17)) including diffusional and potential components provides natural extension of the one-dimensional "flux of growth" in the classical theory.

The total number of stable clusters ( = nuclei)

$$
N(t)=N_{0} \iiint_{V^{*}} w(\boldsymbol{\xi}) d^{N} \boldsymbol{\xi},
$$

leads, after differentiation, to nucleation rate

$$
\frac{\dot{N}}{N_{0}}=\frac{d}{d t} \iiint_{V^{*}} w(\boldsymbol{\xi}) d^{N} \boldsymbol{\xi}=\iiint_{V^{*}} \frac{\partial w}{\partial t} d^{N} \boldsymbol{\xi}-\frac{d \mathbf{R}^{*}}{d t} \cdot \iint_{S^{*}} w(\boldsymbol{\xi}) d^{N-1} \mathbf{S},
$$

which, after application of the equation of continuity (Eq. (3.16)) and GaussOstrogradsky theorem reduces to two terms representing generalized thermal and athermal nucleation rates

$$
\frac{\dot{N}}{N_{0}}=-\iint_{S^{*}} j(\boldsymbol{\xi}) d^{N-1} \mathbf{S}-\frac{d \mathbf{R}^{*}}{d t} \cdot \iint_{S^{*}} w(\boldsymbol{\xi}) d^{N-1} \mathbf{S},
$$

$\mathbf{R}^{*}$ is a vector normal to critical hypersurface $S^{*}$. Physical sense of thermal and athermal nucleation rates is the same as in the classical theory. The first term in Eq. (3.24) presents thermally activated flux of clusters through critical hypersurface $S^{*}$. What is new in the generalized theory is variety of ways (mechanisms) in which an unstable cluster may reach status of a stable nucleus.

Potential components of the flux $\mathbf{j}(\boldsymbol{\xi})$ include anisotropic growth in $n$ dimensions, $j_{\text {growth }}$, rotation in the orienting field, $j_{\text {rot }}$, translation with respect to field poles, $j_{\text {trans }}$, and transformation of internal structure (e.g. healing of defects)

$$
\mathbf{j}(\boldsymbol{\xi})=\left\{j_{\text {growth }}, j_{\text {rot }}, j_{\text {trans }}, j_{\text {heal }}\right\}
$$


It is evident that the generalized theory introduces new mechanisms of nucleation. Figure 17 explains rotational nucleation in a simple two-dimensional space including cluster size, $g$, and orientation, $\vartheta$. The sinusoidal curve $g^{*}(\vartheta)$ represents hypersurface $S^{*}$ separating region of stability from the rest of space. To reach the region of stability and cross $S^{*}$ - small cluster can grow, changing its size, $g$, or rotate reducing orientation angle $\vartheta$ without changing its size. Both mechanisms are driven with the appropriate gradients of free energy

$$
j_{\text {grow }}=-D_{\text {grow }}\left[\frac{\partial w(g, \vartheta)}{\partial g}+\frac{w}{k T} \frac{\partial \Delta F(g, \vartheta)}{\partial g}\right],
$$

and

$$
j_{\text {rot }}=-D_{\text {rot }}\left[\frac{\partial w(g, \vartheta)}{\partial \vartheta}+\frac{w}{k T} \frac{\partial \Delta F(g, \vartheta)}{\partial \vartheta}\right] .
$$

In a similar way, one can define translational nucleation, $j_{\text {trans }}$, resulting from motion of the cluster in a gravitational or electrostatic field.

$$
j_{\text {trans }}=-D_{\text {trans }}\left[\frac{\partial w}{\partial x}+\frac{w}{k T} \frac{\partial \Delta F}{\partial x}\right]
$$

An extension of the concept of thermal nucleation is nucleation resulting from healing of internal structure by elimination of defects described with the variable $\sigma$

$$
j_{\text {heal }}=-D_{\text {heal }}\left[\frac{\partial w}{\partial \sigma}+\frac{w}{k T} \frac{\partial \Delta F}{\partial \sigma}\right] .
$$

All the above mechanisms involve thermally activated motions characterized by the diffusion tensors: $D_{\text {grow }}$ is controlled by diffusion of single kinetic elements to and from cluster surface; $D_{\text {rot }}$ and $D_{\text {trans }}$ are, respectively, rotational and translational diffusion coefficients of the entire clusters and thus require much higher activation energies. On the other hand, diffusion coefficient involved in healing of internal structure as associated with motions of small defects (vacancies, kinks, etc.) is comparable to $D_{\text {grow }}$.

$$
D_{\text {rot }} \ll D_{\text {grow }} ; D_{\text {trans }} \ll D_{\text {grow }} ; D_{\text {heal }} \approx D_{\text {grow }}
$$

For this reason it is expected that rotational and translational nucleation rates are negligibly small compared to the classical growth mechanism, unless strong orienting (gravitational) fields are applied. This does not deny great importance of external fields in determining critical nucleation conditions, selectivity of the transition and athermal effects. As far as diffusion coefficients are concerned, healing nucleation rates should be comparable with growth controlled mechanism. 
a)

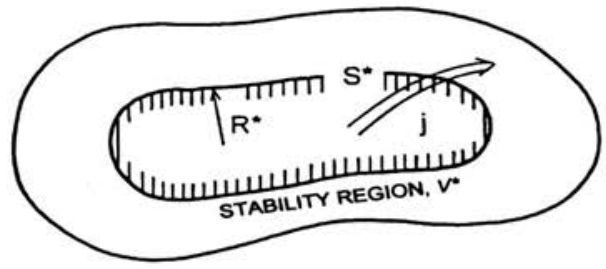

b)

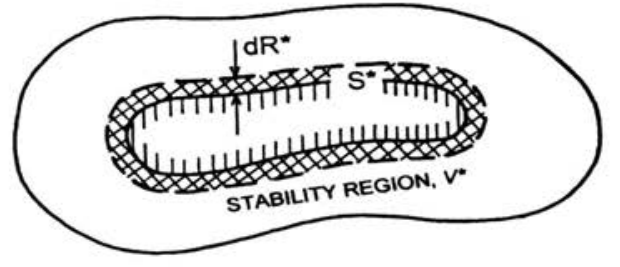

Figure 18. Two basic mechanisms of nucleation a) thermal nucleation - flux of unstable clusters into the region of stability $V^{*}$. b) athermal nucleation - embedding of unstable clusters by the expanding region of stability $V^{*}$.

Thermal nucleation mechanism is presented schematically in Fig. 18(a) as a flux into the stability region $V^{*}$ over the the potential barrier distributed along the critical surface $S^{*}$.

Athermal nucleation consists in non-activated promotion of unstable clusters to the status of stable nuclei by expansion of the region of stability, $V^{*}$ (Fig. 18(b)). Clusters originally contained in the unstable region change their status without changing their configuration. Athermal process follows changes of external conditions (temperature $T$, pressure $p$, etc.) and disappears in constant ("isothermal") conditions. In a full analogy to critical cluster size $g^{*}$ in the classical theory (Eq. (3.9)), critical surface radius $\mathbf{R}^{*}$ follows changes of external conditions

$$
\frac{d \mathbf{R}^{*}}{d t}=\frac{\partial \mathbf{R}^{*}}{\partial T} \frac{d T}{d t}+\frac{\partial \mathbf{R}^{*}}{\partial p} \frac{d p}{d t}+\ldots
$$

The generalized theory of nucleation provides a powerful tool for solving many specific problems. It predicts new phenomena not studied yet (gravitational effects, nucleation by healing) and reduces to the classical concepts of Volmer, Weber, Frenkel and Turnbull.

\section{References}

\section{References for Sec. 1}

1. H. Mark and A.V. ToBolsky, Physical Chemistry of High Polymeric Systems, Interscience, New York, 1950. 
2. M.V. Volkenstein, Configurational Statistics of Polymer Chains, Interscience, New York, 1963.

3. P.J. Flory, Statistical Mechanics of Chain Molecules, Interscience, New York, 1969.

4. H. Yamakawa, Modern Theory of Polymer Solutions, Harper\&Row, New York, 1971.

5. A. Ziabicki, L. Jarecki, A. Perico, and M. Guenza, Macromol. Theory Simul., Vol.4, 643, 1995.

6. O. Gerngross and C. Hermann, Z. Physik. Chemie, Vol.B10, 371, 1930.

7. O. Kratky and H. Mark, Z. Physik. Chemie, Vol.B36, 129, 1937.

8. A. Keller, Makromol. Chemie, Vol.34, 1, 1959.

9. F. P. Price, J.Polymer Sci., Vol.42, 49, 1960.

10. A. Peterlin, E.W. Fischer, and C. Reinhold, J.Chem. Phys., Vol.37, 1403, 1962.

\section{References for Sec. 2}

11. W. KuHn and F. Grün, Kolloid Z., Vol.101, 248, 1942.

12. P.J. Flory, Principles of Polymer Chemistry, Cornell University Press, Ithaca NY, 1953.

13. J. WALASEK, PhD Thesis, IFTR Warsaw, 1976.

14. A.V. Tobolsky, D.J. Metz., and R.B. Mesrobian, J. Am. Chem. Soc., Vol.72, 1942, 1950.

15. L.C. Case, J. Polymer Sci., Vol.45, 397, 1960.

16. J. Scanlan, J. Polymer Sci., Vol.43, 501, 1960.

17. L.Mullins and A.G. Thomas, J. Polymer Sci., Vol.43, 13, 1960.

18. H. Jacobson and W.H. Stockmayer, J. Chem. Phys., Vol.18, 1600, 1950.

19. E. Helfand and A. Tonelli, Macromolecules, Vol.7, 59, 832, 1974.

20. A. ZiABICKI and J. Walasek, Macromolecules, Vol.11, 471, 1978.

21. A. Ziabicki, Polymer, Vol.20, 1373, 1979.

22. A. ZiABicki and J. Walasek, (in:) Biological and Synthetic Polymer Networks, O. Kramer, (Ed.), Elsevier, pp.517-530, 1987.

23. A. Ziabicki and J. Walasek, Colloid \& Polymer Sci., Vol.266, 114, 1988.

24. M.S. Green and A.V. Tobolsky, J. Chem. Phys, Vol.14, 80, 1946.

25. M. Yамамото, J. Phys. Soc. Japan, Vol.11, 413, 1956.

26. M. Kość and A. ZıABICKI, Macromolecules, Vol.15, 1507, 1982.

27. F. Bueche, J. Chem. Phys., Vol.20, 1959, 1952; ibid. Vol.25, 599, 1956.

28. A. Ziabicki and R. Takserman-Krozer, J. Polymer Sci.,Ser. A2, Vol.7, 2005, 1969.

29. A. Ziabicki, J. Chem. Phys, Vol.64, 4100, 41007, 1976. 
30. D. Acierno and A. Ziabicki, J. Chem. Phys., Vol.64, 4100, 4107, 1976.

31. A. Ziabicki, Colloid \& Polymer Sci., Vol.254, 1, 1976.

32. R. Takserman-Krozer and A. Ziabicki, J. Polymer Sci.,Ser. A2., Vol.8, 321, 1970.

33. A. Ziabicki and R. Takserman-Krozer, J. Polymer Sci.,Ser. A., Vol.7, 2005, 1969.

34. A. ZIABICKI (in:) Problémes de Rhéologie et de Mécanique des Sols, PWN, Warszawa, pp.501-513, 1977.

\section{References for Sec. 3}

35. A.N. Kolmogoroff, Izvestiya Akad. Nauk SSSR, Ser. Math., Vol.3, 335, 1937.

36. M. Avrami J. Chem. Phys., Vol.7, 1103, 1939.

37. W.A. Johnson and R.F. Mehl, Trans. AIME, Vol.135, 416, 1939.

38. U.R. Evans, Trans. Faraday Soc., Vol.41, 365, 1945.

39. M. Volmer and A. Weber, Z. Phys. Chemie, Vol.119, 277, 1926.

40. R. Becker and W. Döring, Ann. Phys., Vol.24, 719, 1935; Vol.32, 128, 1938.

41. J. Frenkel, Kinetic Theory of Liquids, Oxford University Press, London, 1946.

42. D. Turnbull and J.C. Fisher, J. Chem. Phys., Vol.17, 71, 1949.

43. A. Ziabicki J. Chem. Phys., Vol.48, 4368, 4374, 1967.

44. A. Ziabicki J. Chem. Phys., Vol.85, 3042, 1985.

45. A. ZiABICKI, Multidimensional Theory of Crystal Nucleation, (in:) Mathematical Modelling for Polymer Processing, V. Capasso, (Ed.), Springer Verlag, 2003.

46. J.C. Fisher, J.H. Hollomon, and D. Turnbull, J. Appl. Phys., Vol.19, 775, 1948.

47. L. Mandelkern, Crystallization of Polymers, McGraw \& Hill, New York, 1964.

48. J.I. Lauritzen and J.D.Hoffman, J. Res. NBS, Vol.64A, 73, 1960.

49. A. Ziabicki and L. JARECKi, J. Chem. Phys., Vol.101, 2267, 1994.

50. A. ZiABICKI and L. JARECKI, Macromol. Symposia, Vol.90, 31, 1994.

51. A. ZIABICKI, Arch. Mechanics, Vol.42, 703, 1990.

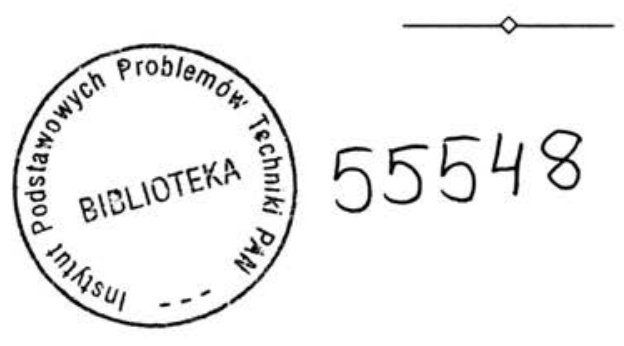

